Journal of

Women's Health and Gynecology

\title{
Is There Any Association Between the Angiogenic Factors Sflt-1 / Plgf And Intra- uterine Growth Restriction in Patients with Preeclamsia?
}

\section{Ammar Al Naimi*, Monica Schmidt-Fittschen, Akos Herzeg, Ioannis Kyvernitakis, Frank Louwen and Franz Bahlmann}

Department of Obstetrics and Gynecology, Buergerhospital Frankfurt, Goethe-University of Frankfurt, Germany

*Corresponding author: Dr. Ammar Al Naimi, Department of Obstetrics and Gynecology, Buergerhospital - Dr. Senckenbergische Stiftung, Nibelungenallee 37-41, D-60318 Frankfurt am Main, Tel: +0049-69-1500-1509, Fax: 0049-69-1500-400, E-mail: ammar.alnaimi@uclmail.net

Received Date: May 21, 2019 Accepted Date: June 17, 2019 Published Date: June 19, 2019

Citation: Al Naimi (2019) Is There Any Association Between the Angiogenic Factors Sflt-1 / Plgf And Intrauterine Growth Restriction in Patients with Preeclamsia? J Womens Health Gyn 6: 1-7.

\begin{abstract}
Purpose: To evaluate the association between the soluble FMS-like tyrosin kinase-1 (sFlt-1) and placental growth factor (PlGF) with intrauterine growth restriction (IUGR) in women with preeclampsia. The correlation between PlGF, sFlt-1 and IUGR (which is confirmed at delivery) and the value of adding the Doppler ultrasound findings of the uterine artery to this combination was evaluated.
\end{abstract}

Results: This single-center prospective observational cohort study included 728 participants diagnosed with clinical preeclampsia of which $80 \%$ (584 patients) had complete follow-up till delivery at our center while 19\% (138) delivered a baby with birth weight below the 10th percentile. These patients had significantly higher sFlt-1 as well as lower PlGF concentrations in comparison to patients without IUGR. The delta in PlGF concentration was more sensitive than that of sFlt-1 (Wilcoxon-Mann-Whitney $\mathrm{r}=0.46, \mathrm{r}=0.28$ ). sFlt-1/PlGF ratio could predict IUGR with a $79.1 \%$ sensitivity and a $72.4 \%$ specificity. Logistic regression showed that the combination of sFlt-1/PlGF with uterine artery notching slightly improves the predictive value for IUGR from the isolated factors on their own with $79.5 \%$ sensitivity. PI of the uterine artery was not beneficial in improving the predictive power of this combination. Wald's p-values are 0.000012 for s-Flt-1/PlGF, 0.000285 for notching and 0.04945 for PI of the uterine artery.

Conclusions: Angiogenesis factors sFlt-1/PlGF are beneficial for predicting IUGR in high-risk groups such as a population with preeclampsia. Their combination with uterine artery notching could provide valid models for IUGR screening.

Keywords: IUGR, sFlt-1, PlGF, Angiogenic Factors, Uterine Artery Doppler

(C)2019 The Authors. Published by the JScholar under the terms of the Creative Commons Attribution License http://creativecommons.org/licenses/ by/3.0/, which permits unrestricted use, provided the original author and source are credited. 


\section{Introduction}

The spiral arteries undergo remodeling throughout pregnancy that is essential for normal fetal growth. This remodeling increases the diameter of the end spiral arteries by 4-6 times as well as the blood flow from initial $50 \mathrm{ml} / \mathrm{min}$ to $600 \mathrm{ml} /$ min [1]. Failed remodeling is associated with increased risk for intrauterine growth restriction (IUGR) [2]. IUGR is a failure of the fetus to reach its genetic growth potential and is confirmed as weight below the 10th percentile for the gestational age accompanied by an increased impedance of the umbilical artery [3]. It commonly complicates pregnancy and increases the risk for adverse outcome such as stillbirth, neonatal death and increases perinatal morbidity. Other cases of small for gestational age (SGA) are associated with congenital anomalies, infections and drugs [4].

The pathophysiology of IUGR, similar to preeclampsia, is based on the failure of the spiral arteries to physiologically transform during pregnancy from high pressure, low capacity vessels to low pressure high capacity ones [5]. Placental growth factor (PlGF) and soluble FMS-like tyrosine kinase-1 (sFlt-1) are two important factors in regulating this transformation. PlGF is a proangiogenic growth factor that stimulates cellular proliferation and is expressed in the syncytiotrophoblast [6], while sFlt1 is a truncated splice of the vascular endothelial growth factor (VEGF) receptor that binds to both PIGF and VEGF and block them, thus antagonising their action. It is mainly produced in the syncytiotrophoblasts and monocytes [7].

Several publications show the importance of sFlt-1/ PlGF in the development of preeclampsia, it's prediction and confirming the diagnosis [8]. sFlt-1 is higher in isolated IUGR pregnancies than in normal pregnancies, and its level of correlates directly with the abnormality in the uterine and umbilical Doppler impedance [9]. Failed remodeling causes abnormalities in the uterine artery Doppler with increased impedance and diastolic notching. The uterine artery Doppler velocimetry can be used in screening for patients at risk for developing IUGR [10]. Antenatal diagnosis of IUGR has low sensitivity with a detection rate of $25 \%$ in a high-risk population [11] and only $15 \%$ in lowrisk population [12]. Newer data report better detection rates, but they are still lacking with an overall detection rate of $36 \%$ generally and $56 \%$ in a high-risk population [13].

Evaluation of the uterine artery Doppler in the first and second trimester could help in screening for early-onset IUGR associated with preeclampsia. Reported detection rates vary from $30 \%$ [14] to about $75 \%$ which could also be improved to reach $90 \%$ when combined with other maternal risk factors and biochemical markers [15].

This study assesses the value of the angiogenic factors for predicting IUGR in a population with manifested preeclampsia, and to assess the value of combining uterine artery Doppler with the angiogenic factors for improving the predictive power for IUGR.

\section{Materials and Methods}

This was a single center prospective observational cohort study and was conducted at Buergerhospital (Frankfurt am Main, Germany). We included 728 singleton pregnancies in this study after giving consent. They were selected from a high-risk collective with manifested preeclampsia. The collective was classified as high-risk according to the German antenatal care guidelines. Additional risk factors including maternal age, history of IUGR, increased Body Mass Index (BMI), chronic hypertension, autoimmune diseases etc. were separately recorded. The data from this collective was analyzed for assessing the value of the angiogenic factors in predicting IUGR knowing that these patients have manifested preeclampsia. Exclusion criteria included complications that would affect the course of the pregnancy, such as chromosomal or structural anomalies, premature preterm rupture of membranes, placenta praevia, and breech presentation. Medical history, obstetric history, ultrasound examination and laboratory results were recorded for each patient.

Ultrasound Doppler sonography was utilized for diagnosing notching of the uterine arteries, mean PI of the uterine arteries, and IUGR. Biparietal diameter, abdominal circumference, and femur length were used for estimating fetal weight. The ultrasound examinations and the Doppler investigations were performed using the Aplio 500 and Aplio 300 (Toshiba Medical System Europe) and GE Voluson E8 and GE Voluson 730 Expert (GE Healthcare) with 5.0-MHz convex transducers.

Blood samples were taken to assess PlGF, sFlt-1, and their ratio. The serum levels of sFlt-1 and PlGF were measured in Synlab Leinfelden, using the immunoassay kits (Elecsys ${ }^{\circledR}$ sFlt-1 and Elecsys ${ }^{\circledast} \mathrm{PlGF}$ ) manufactured by Roche Diagnostics $\mathrm{GmbH}$.

Pregnancy outcome for the patients who were not lost to follow up was recorded with mode of delivery, gestational age at delivery, 5 ' minute APGAR score and fetal weight in gram (g). IUGR suspicion was recorded based on the ultrasound findings, but was only confirmed when birth weight was below the tenth percentile for the gestational age at delivery. 
Statistical analysis was undertaken with the help of Biometrische Analyse von Stichproben, BiAS (biometric analysis of random samples) version 10.12 from Dr. Ackermann, Goethe University Frankfurt am Main, Germany. The Wilcoxon-Mann-Whitney-U, Chi square contingency table, Pearson's contingency coefficient (C), Receiver operating characteristic (ROC) curves, Logistic regression and the Kruskal-Wallis test were utilized in the analysis. A p-value of 0.05 was set as the cut-off point for significance.

\section{Results}

This study included 728 individual patients, which is a fraction of the 10600 deliveries in this hospital for this time period. The chi-square test was used to evaluate the prenatal diagnosis of IUGR with postnatally confirmed IUGR cases. The results showed good diagnostic power with $86.2 \%$ sensitivity and $93 \%$ specificity $(\mathrm{p}<10-6)$.

Follow-up data with delivery information at the study hospital were available for 584 patients, 138 of which (23.6\%) had IUGR at delivery. sFlt-1, PlGF and their ratio were separately tested against IUGR-related delivery. Table 1 summarizes the results of Wilcoxon-Mann-Whitney-U test for the angiogenic factors in relation to IUGR-related delivery. sFlt-1 showed a weak to moderate effect for predicting IUGR-related delivery with $\mathrm{r}$ of 0.29 . PlGF and the ratio showed moderately good effect with high statistical significance.
Roc curve analyses were utilized for assessing the sensitivity and specificity of the angiogenic factors for predicting IUGR at delivery. The optimal cut-off point for sFlt-1 was $7077 \mathrm{pg} / \mathrm{ml}$ with a $63.2 \%$ sensitivity, $69.6 \%$ specificity and $33.6 \%$ misclassification rate. The optimal cut-off point for PlGF was $93.5 \mathrm{pg} / \mathrm{ml}$ with a $74.6 \%$ sensitivity, $76.5 \%$ specificity and $24.4 \%$ misclassification rate. The optimal cut-off point for the ratio was 69.39 with a $79.1 \%$ sensitivity, $72.4 \%$ specificity and $24.2 \%$ misclassification rate.

Spearman's test was used to assess the correlation between sFlt-1, PlGF and their ratio on the one side with PI of the uterine artery, diabetic status, birth weight, 5 minutes APGAR score, and gestational age at delivery on the other side. The statistically significant correlations ranged mainly from poor to moderate. Uterine artery PI showed a moderately good negative correlation with PlGF. Birth weight showed a moderately good positive correlation with PlGF and a negative correlation with the quotient. The results of these tests are summarized in table 2

\begin{tabular}{|l|l|l|l|l|l|l|l|}
\hline & IUGR related & median & min. & max. & n. & r & P \\
\hline sFlt-1 & no & 4217 & 22.97 & 69761 & 441 & 0.29 & 0.69 \\
\hline & yes & 8239 & 85 & 45017 & 136 & & \\
\hline PlGF & no & 177.5 & 7 & 3604 & 441 & 0.46 & 0.19 \\
\hline & yes & 56.11 & 3 & 528 & 136 & & \\
\hline Ratio & no & 24.92 & 0.45 & 723.4 & 441 & 0.45 & 0.81 \\
\hline & yes & 154.4 & 2.55 & 1551.3 & 136 & & \\
\hline
\end{tabular}

Table 1. The results of the Wilcoxon-Mann-Whitney-U test for the angiogenesis factors and IUGR at delivery (min: minimum, max: maximum, n: number of patients, r: effect size, P: Mann-Whitney P value)
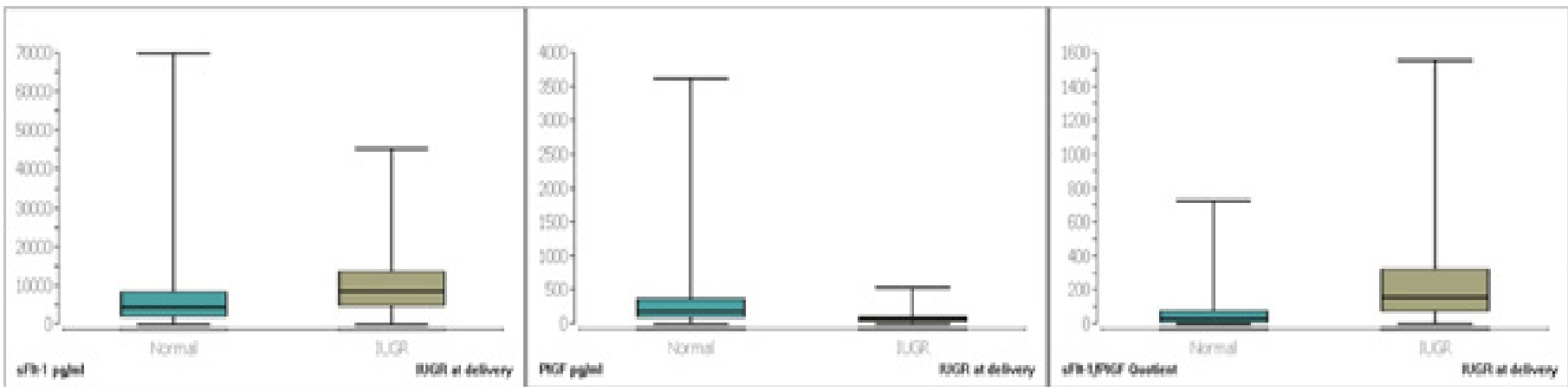

Figure 1. Box plot of sFlt-1, PlGF, and their ratio for patients who had IUGR-related delivery against isolated preeclampsia. 

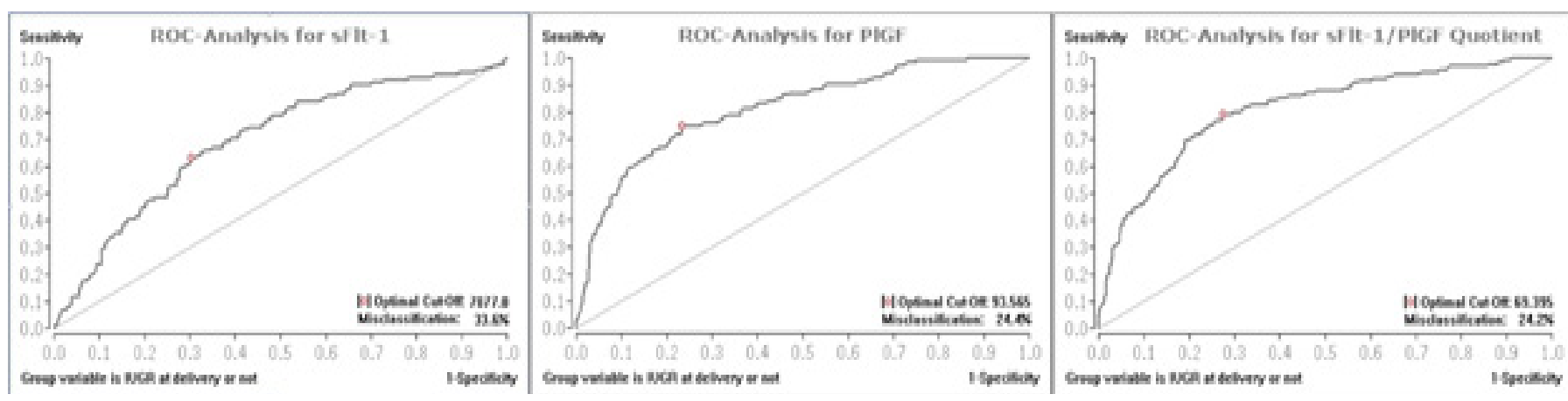

Figure 2. Roc curve analysis for sFlt-1, PlGF, and their ratio as a diagnostic value for predicting IUGR at delivery.

\begin{tabular}{|c|c|c|c|c|}
\hline & & sFlt-1 & PlGF & Ratio \\
\hline Uterine artery PI & $\rho$ & 0.14 & -0.43 & 0.33 \\
\hline & $\mathrm{p}$ & $<10-3$ & $<10-6$ & -0.03 \\
\hline Diabetes & $\rho$ & -0.07 & -0.04 & 0.47 \\
\hline $\begin{array}{c}\text { Gestational age at } \\
\text { delivery }\end{array}$ & $\mathrm{p}$ & 0.06 & 0.25 & -0.39 \\
\hline 5 minutes APGAR & $\mathrm{p}$ & -0.32 & 0.3 & $<10-6$ \\
\hline & $\rho$ & $<10-6$ & $<10-6$ & -0.22 \\
\hline Birth weight & $\mathrm{p}$ & -0.15 & 0.23 & $<10-6$ \\
\hline & $\rho$ & $<10-3$ & $<10-6$ & -0.49 \\
\hline
\end{tabular}

Table 2. The results of Spearman's correlation between the angiogenesis factors and Doppler results, diabetes and other delivery variables ( $\rho=$ Spearman's correlation Rho, $p=$ value for statistical significance)

Table 3. shows the range, median and the quartiles of the uterine artery PI in patients who developed IUGR in comparison to those without. Wilcoxon-Mann-Whitney-U test showed a statistically significant $(\mathrm{p}<10-6)$ moderate to strong effect of the uterine artery PI for predicting IUGR with $r$ of 0.4 and Mann-Whitney P of 0.75 . This correlation is also shown as a box plot in figure 3 .

Chi-square test for notching showed a positive correlation with IUGR-related delivery. Corrected Pearson's contingency coefficient for this correlation was $0.58(\mathrm{P}<10-6)$.
Logistic regression was used to test a model of using notching and PI of the uterine artery in combination with the sFlt-1/PlGF ratio for anticipating IUGR-related delivery. The results show that the addition of the uterine artery PI to the combination of notching and the ratio does not improve the diagnostic power. The diagnostic power of the combination of notching and sFlt-1/PlGF was $79.5 \%$. Wald's $\mathrm{P}$ value for the quotient is $10-5$, for notching $3 \times 10-4$.

\begin{tabular}{|l|l|l|l|l|}
\hline Uterine artery PI & median & Range & 1. quartile & 3. quartile \\
\hline Normal & 0.77 & $0.37-2.99$ & 0.6 & 1.07 \\
\hline IUGR & 1.23 & $0.33-3.33$ & 0.94 & 1.66 \\
\hline
\end{tabular}

Table 3. Comparison of the uterine artery PI values in patients who develop IUGR and normal patients 


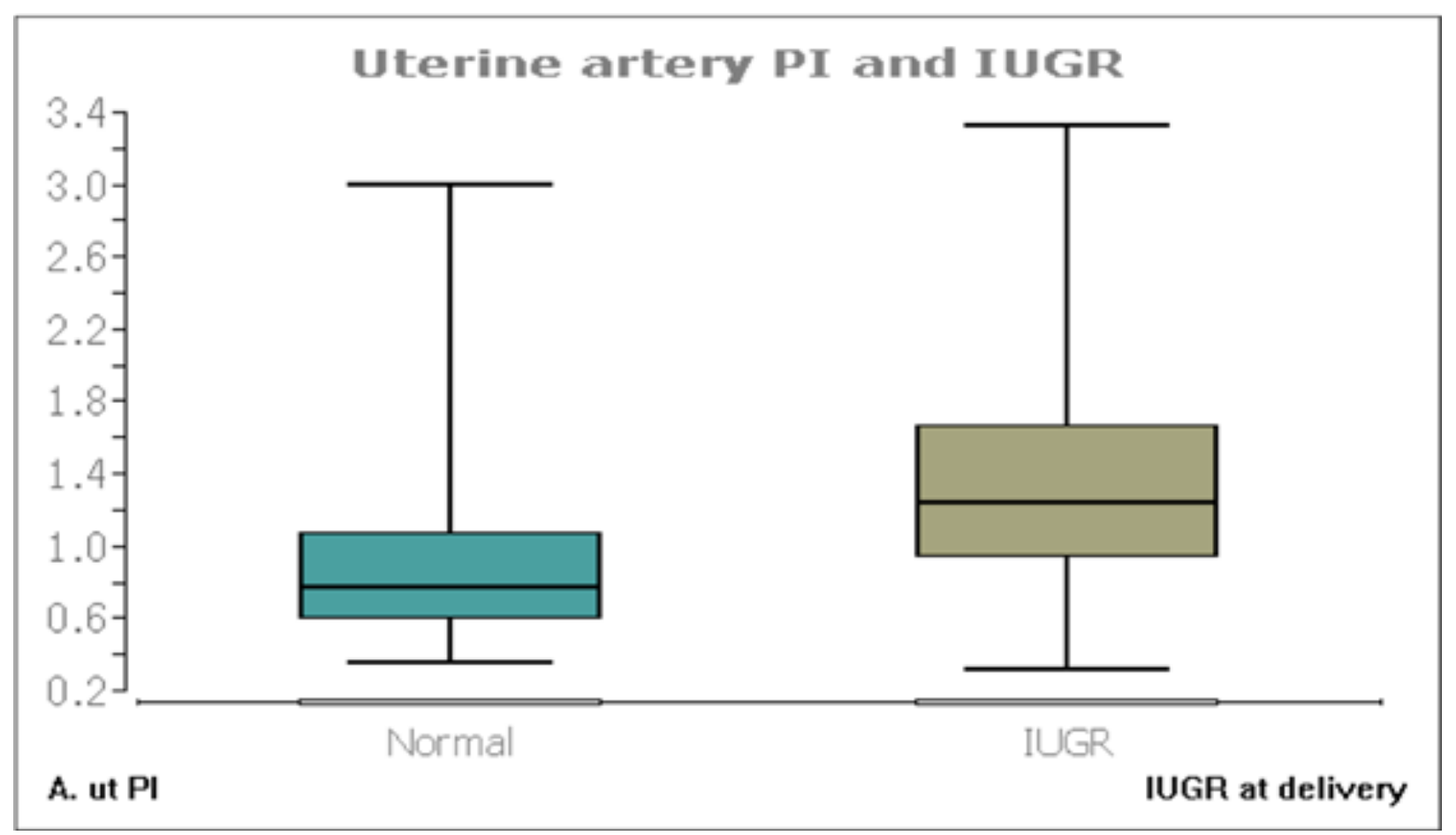

Figure 3. Box plot of the uterine artery PI for patients who eventually develop IUGR against those without.

\section{Discussion}

We made the observation that patients who eventually had IUGR had higher levels of sFlt-1, lower levels of PlGF and higher sFlt-1/PlGF ratios than in isolated preeclampsia patients. sFlt-1 in IUGR pregnancies versus isolated preeclampsia had medians of $8239 \mathrm{pg} / \mathrm{ml}$ and $4217 \mathrm{pg} / \mathrm{ml}$ respectively and a statistically significant weak to moderate Wilcoxon Mann Whitney-U effect of 0.29 . Using an sFlt-1 cut-off value of $7077 \mathrm{pg} / \mathrm{ml}$ could be used for anticipating IUGR-related delivery with $63.2 \%$ sensitivity, $69.6 \%$ specificity and $33.6 \%$ misclassification rate (false positive + false negative). This weak performance might be the reason why some studies suggest that there was no sFlt-1 increase in IUGR pregnancies, especially publications with small sample size that might miss the weak to moderate increase $[16,17]$. The PlGF medians were $177.5 \mathrm{pg} / \mathrm{ml}$ and $56.11 \mathrm{pg} / \mathrm{ml}$ respectively with moderate Wilcoxon Mann Whitney-U effect of 0.46 . The best cut-off value of $93.5 \mathrm{pg} / \mathrm{ml}$ for PlGF could be used for predicting IUGR in preeclampsia patients with $74.6 \%$ sensitivity, $76.5 \%$ specificity and $24.4 \%$ misclassification rate. This is a better performance than sFlt-1, which might provide a reason for using PlGF in screening for pregnancies with risk for IUGR. sFlt-1/PlGF ratios had medians of 154.4 vs 24.92 respectively and statistically significant moderately good Wilcoxon Mann Whitney-U effect of 0.45 . Using a ratio cut-off value of 69.39 could be used for anticipating IUGR with a $79.1 \%$ sensitivity, $72.4 \%$ specificity and $24.2 \%$ misclassification rate, which is a comparable performance to PlGF. We analyzed our population and correlated between severe and moderate preeclampsia among those with and without IUGR and found a difference with no statistical significance $(p=0,25)$. This finding supports the theory that the difference of sFlt-1/PlGF between IUGR and non-IUGR cases was not caused by the severity of preeclampsia.

sFlt-1 correlates indirectly, PlGF directly, and sFlt-1/ PlGF ratio indirectly, with gestational age at delivery, neonatal birth weight and very weakly with the 5 minutes APGAR. The correlation coefficients of the ratio are stronger than sFlt- 1 and PlGF on their own, which renders it useful for anticipating adverse fetal outcome at delivery.

The incidence of IUGR in our population was $1.3 \%$, which is lower than the quoted global IUGR incidence of $2.8 \%$ [18]. IUGR has high correlation with abnormal Doppler findings, stillbirths, iatrogenic prematurity, fetal distress and caesarean sections [19]. Our data mirrors this correlation.

IUGR patients delivered significantly earlier than those with isolated preeclampsia with average gestational ages of 34/1 and 37/4 weeks respectively, and had significantly smaller babies. The earlier delivery can definitely be attributed to iatrogenic causes. The combination of early delivery and lighter 
birth weight might be a reason for adverse outcome. IUGR cases were associated with higher rates of cesarean section $73 \%$ in comparison to $50 \%$ in the isolated preeclampsia collective.

Intrauterine fetal demise (IUFD) had a significant association with extreme cases of IUGR, which is to be expected as reported in literature [20]. Identified IUGR cases have a 5- to 10 -fold risk for developing IUFD. These need to be closely monitored with methods such as umbilical artery Doppler, which leads to a reduction of the IUFD rate and increases iatrogenic prematurity as in our data without increasing the neonatal mortality [21].

There is a moderate negative correlation between PlGF and the uterine artery PI with Spearman's $\rho$ value of -0.43 . Both notching and PI of the uterine artery correlate with predicting IUGR. Combining sFlt-1/PlGF with notching provides an effective model with a diagnostic power of $79.5 \%$ for anticipating IUGR without the need for uterine artery PI.

These results show that using the angiogenic factors in combination with the ultrasound findings could provide valid models for predicting IUGR in preeclampsia population. This is a reconfirmation of preexisting data regarding the relationship between sFlt-1, PlGF and IUGR in a normal population, and this data is specifically within the subpopulation of manifested preeclampsia.

\section{Compliance with Ethical Standards}

Conflict of interest disclosures: Al Naimi, SchmidtFittschen, Herzeg, Kyvernitakis, Louwen and Bahlmann declare that we have no conflict of interest

Ethical approval: This work has been approved by the internal Ethical Board of Buergerhospital, which is led by Professor Schwenn. All procedures performed in studies involving human participants were in accordance with the ethical standards of the institutional and/or national research committee and with the 1964 Helsinki declaration and its later amendments or comparable ethical standards.

Informed consent: Informed consent was obtained from all individual participants included in the study according to the declaration of Helsinki and the German law.

\section{Acknowledgments}

This work was supported by the Dr. Senckenberg Foun-

\section{References}

1. Zhou Y, Fisher SJ, Janatpour M, Genbacev O, Dejana E, Wheelock M et.al (1997) Human cytotrophoblasts adopt a vascular phenotype as they differentiate. A strategy for successful endovascular invasion? J Clin Invest 99:2139-2151.

2. Carbillon L, Challier JC, Alouini S, Uzan M and Uzan S. (2001) Uteroplacental circulation development: Doppler assessment and clinical importance. Placenta 22:795-799.

3. Alexander GR, Himes JH, Kaufman RB, Mor J and Kogan M. A. (1996) United States national reference for fetal growth. Obstet Gynecol 87:163-168.

4. Jacobsson B, Ahlin K, Francis A, Hagberg G, Hagberg $\mathrm{H}$ and Gardosi J. (2008) Cerebral palsy and restricted growth status at birth: population-based case-control study. BJOG 115:1250-1255.

5. Kaufmann P, Black S and Huppertz B. (2003) Endovascular trophoblast invasion: implications for the pathogenesis of intrauterine growth retardation and preeclampsia. Biol Reprod 69:1-7.

6. Clark DE, Smith SK, Licence D, Evans AL and Charnock-Jones DS. (1998) Comparison of expression patterns for placenta growth factor, vascular endothelial growth factor (VEGF), VEGF-B and VEGF-C in the human placenta throughout gestation. J Endocrinol 159:459-467.

7. Rajakumar A, Brandon HM, Daftary A, Ness R and Conrad KP. (2004) Evidence for the functional activity of hypoxia-inducible transcription factors overexpressed in preeclamptic placentae. Placenta 25:763-769.

8. Bahlmann F and Al Naimi A. (2016) Using the angiogenic factors sFlt-1 and PlGF with Doppler ultrasound of the uterine artery for confirming preeclampsia. Arch Gynecol Obstet 294:1133-1139.

9. Chaiworapongsa T, Espinoza J, Gotsch F, Kim YM, Kim GJ, Goncalves LF, et.al. (2008) The maternal plasma soluble vascular endothelial growth factor receptor-1 concentration is elevated in SGA and the magnitude of the increase relates to Doppler abnormalities in the maternal and fetal circulation. J Matern Fetal Neonatal Med 21:25-40.

10. Papageorghiou AT, Yu CK, Bindra R, Pandis G and Nicolaides KH. (2001) Fetal Medicine Foundation Second Tridation, Frankfurt am Main. 
mester Screening Group. Multicenter screening for pre-eclampsia and fetal growth restriction by transvaginal uterine artery Doppler at 23 weeks of gestation. Ultrasound Obstet Gynecol 18:441-449.

11. Hepburn M and Rosenberg K. (1986) An audit of the detection and management of small-for-gestational age babies. Br J Obstet Gynaecol 93:212-216.

12. Backe B and Nakling J. (1993) Effectiveness of antenatal care: a population-based study. Br J Obstet Gynaecol 100:727732 .

13. Dougan C, Bell S, Mc Afee C, Armstrong N and Hunter A. (2014) Prospective audit of antenatal patients in a tertiary obstetric unit to determine antenatal detection rate of Small for Gestation Age (SGA) babies. Arch Dis Child Fetal Neonatal Ed 99:1 A83-A85.

14. Chien PF, Arnott N, Gordon A, Owen P and Khan KS. (2000) How useful is uterine artery Doppler flow velocimetry in the prediction of pre-eclampsia, intrauterine growth retardation and perinatal death? An overview. BJOG 107:196-208.

15. Foidart JM, Munaut C, Chantraine F, Akolekar R and Nicolaides KH. (2010) Maternal plasma soluble endoglin at 1113 weeks' gestation in pre-eclampsia. Ultrasound Obstet Gynecol 35:680-687.

16. Shibata E, Rajakumar A, Powers RW, Larkin RW, Gilmour C, Bodnar LM, et.al. (2005) Soluble fms-like tyrosine kinase 1 is increased in preeclampsia but not in normotensive pregnancies with small-for-gestational-age neonates: relationship to circulating placental growth factor. J Clin Endocrinol Metab 90:4895-4903.

17. Romero R, Nien JK, Espinoza J, Todem D, Fu W, Chung H, et.al. (2008) A longitudinal study of angiogenic (placental growth factor) and anti-angiogenic (soluble endoglin and soluble vascular endothelial growth factor receptor-1) factors in normal pregnancy and patients destined to develop preeclampsia and deliver a small for gestational age neonate. J Matern Fetal Neonatal Med 21:9-23.

18. Vanlieferinghen S, Bernard JP, Salomon LJ, Chalouhi GE, Russell NE and Ville Y. (2014) Second trimester growth restriction and underlying fetal anomalies. Gynecol Obstet Fertil 42:567-571.
19. McCowan LM, Harding JE and Stewart AW. (2005) Customized birthweight centiles predict SGA pregnancies with perinatal morbidity. BJOG 112:1026-1033.

20. Vergani P, Cozzolino S, Pozzi E, Cuttin MS, Greco M, Ornaghi S et.al. (2008) Identifying the causes of stillbirth: a comparison of four classification systems. Am J Obstet Gynecol 199:319. e1-4.

21. Lindqvist PG and Molin J. (2005) Does antenatal identification of small-for-gestational age fetuses significantly improve their outcome? Ultrasound Obstet Gynecol 25:258-264.

Submit your manuscript to a JScholar journal and benefit from:

ब Convenient online submission

ฯ Rigorous peer review

ฯ Immediate publication on acceptance

q Open access: articles freely available online

9 High visibility within the field

I Better discount for your subsequent articles

Submit your manuscript at http://www.jscholaronline.org/submit-manuscript.php 